\title{
LA EVOLUCION DE LA POBLACION Y DE LA RENTA EN EXTREMADURA
}

\author{
312:339.2 (46 Extremadura)
}

por

\section{Ignacio Ballester Ros}

SUMARIO: I. INTRODUCCION.-II. LA EVOLUCION GENERAL DE LA POBlacion: 1. De la Región en su conjunto y por Provincias. 2. De las capitales de Provincia. 3. De los Partidos Judiciales.-IIl. EL CRECIMIENTO VEGETATIVO Y EL MOVIMIENTO MIGRATORIO.-IV. LA EVOLUCION DE LA POBLACION ACTIVA: 1. EN SU CONJUNTO. 2. POR SECTORES DE LA ACTIVIDAD ECONOMICA.-V. LA EVOLUCION DE LA RENTA: 1. Total. 2. Por habitante.

\section{INTRODUCCION}

Sabida es la importancia que tiene el análisis estadístico regional en todos los países, especialmente en el nuestro, dado que la mayor parte de los fenómenos demográficos, sociales y económicos, no se distribuyen de manera homogénea por el área territorial del país. Las cifras nacionales tienen, de por sí, un valor limitado, si no se tienen en cuenta los valores que alcanzan los fenómenos en las Regiones, permitiendo fijar los contrastes que ofrecen las distintas zonas geográficas.

De ahí el interés del estudio regional. Ya en números anteriores de esta REVISTa hemos comentado las cifras más significativas de dos Regiones situadas en lugares opuestos de nuestra geografía: Cataluña y Galicia; la primera destaca por su potencia demográfica y económica; la segunda, una de nuestras cenicientas, está notoriamente subdesarrollada.

Siguiendo en esta línea, parece oportuno dedicar esta crónica estadística al estudio de la estructura y evolución demográfica, económica y social de Extremadura. Disponemos para ello de cinco 
estudios llevados a cabo por el Consejo Económico-Social de Extiemadura y Huelva, titulados: «Consideraciones sobre la población de Badajoz», "Consideraciones sobre la población de Cáceres», "Migraciones y crecimiento vegetativo en Extremadura», "Evolución de la población activa de Extremadura» y «Evolución de la renta en Extremadura», todos ellos publicados en marzo de 1974.

Por ello, hemos juzgado interesante seguir el análisis de las Regiones, dedicando nuestra atención a una de caracteres tan peculiares como es Extremadura, lo que nos permitirá ir completando la visión general de las Regiones españolas, que tanto interés tiene desde el punto de vista local.

\section{LA EVOLUCION GENERAL DE LA POBLACION}

\section{De la Región en su conjunto y por Provincias}

La población de Extremadura y de cada una de sus Provincias, según los censos de población elaborados por el Instituto Nacional de Estadística, prescindiendo de los de 1857 y 1897, por estar muy próximos (tres años únicamente los separan) a sus inmediatamente posteriores, es:

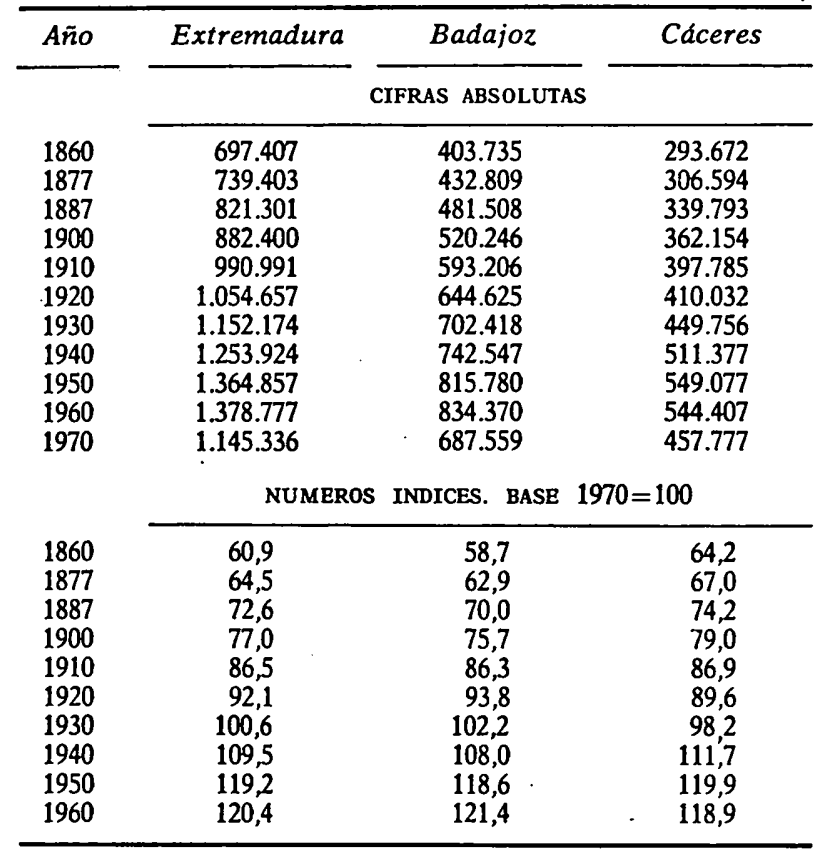


Del examen de las cifras anteriores se deduce, en primer lugar, que la población de Extremadura, que se habia duplicado en cien años, ha disminuido en el último decenio (1960-1970) en una quinta parte. La Provincia de Badajoz presenta un fenómeno paralelo, si bien vio duplicada su población en 1950 (con respecto a la de la fecha de partida), diez años antes que la Región en conjunto, y la pérdida de habitantes en el último decenio ha sido algo superior al promedio regional. La Provincia de Cáceres, por el contrario, no ha llegado a duplicar su población en todo el período, y aunque la pérdida de población en el último decenio ha sido igualmente importante, ofrece una tasa ligeramente inferior.

La Provincia de Badajoz registra un incremento de población con tasas muy dispares; en el período 1860-1900, las tasas, que son bajas, se justifican, en parte, por la emigración a Ultramar, que se hacía patente desde mediados del siglo XIX; las tasas más elevadas corresponden a los decenios 1900-1910 y 1940-1950 y son de igual cuantía: el 10,6 por 100; entre ambos, la población crece a un ritmo superior al registrado hasta principios de siglo, a pesar de la fuerte mortalidad infantil. A partir de 1960 se acusa la intensificación del fenómeno emigratorio hacia el resto de España y al extranjero, a pesar del fuerte crecimiento vegetativo; ya en el decenio 1950-1960, el aumento de su población es muy débil, inferior al 3 por 100; finalmente, la emigración arranca a más de la quinta parte de los habitantes en el último decenio.

La Provincia de Cáceres presenta una tendencia creciente, con tasas más irregulares aún que las correspondientes a la Provincia de Badajoz; el crecimiento máximo corresponde al período 19301940, con el 13,5 por 100; antes de este momento, alternan las tasas débiles de crecimiento por decenios con otras más aceptables. A partir de 1950 disminuye la población, tan sólo un 1 por 100 en el período 1950-1960; pero en el último decenio (1960-1970), al igual que lo ocurrido con Badajoz, la Provincia de Cáceres pierde casi el 19 por 100 de su población, a pesar del incremento vegetativo.

En el decenio 1960-1970, Extremadura ha perdido 233.000 habitantes, lo que resulta realmente asombroso, tratándose del decenio en que se aplicaron los I y II Planes de Desarrollo Económico y Social. Es evidente que de dichos Planes ha quedado totalmente marginada Extremadura. 


\section{De las capitales de Provincia}

La evolución de la población de las ciudades de Badajoz y Cáceres puede deducirse de estas cifras:

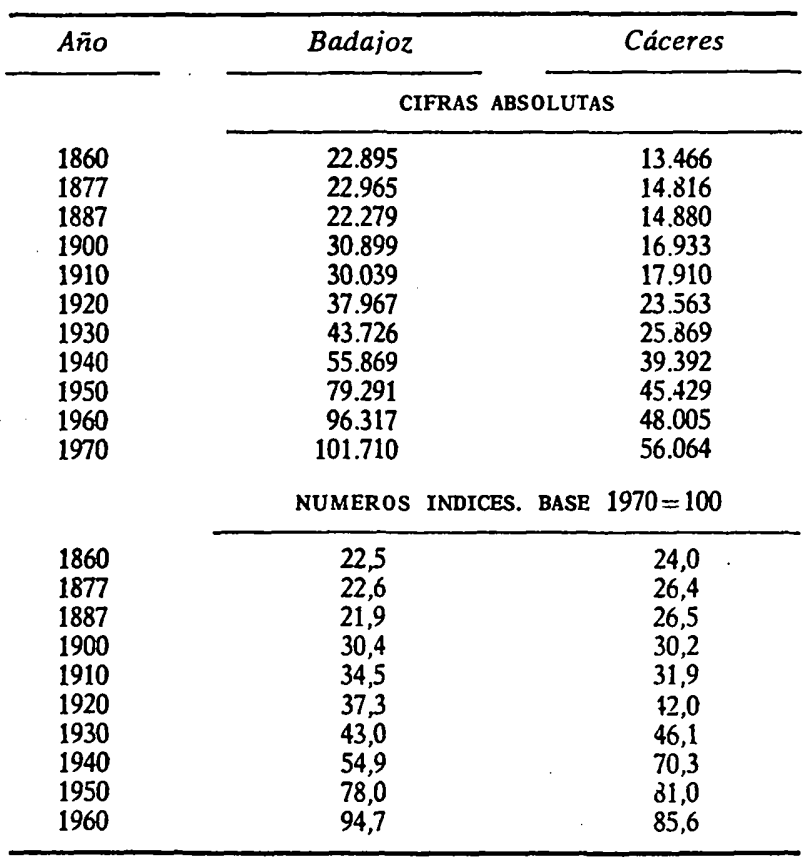

Frente al hecho de que las Provincias de Badajoz y Cáceres han duplicado su población en un siglo, y frente a la tendencia fuertemente descendente en el último decenio, las respectivas capitales, atendiendo al fenómeno general de concentración de la población en las agrupaciones humanas más populosas, ofrecen una tendencia de crecimiento acentuado del número de sus habitantes, que se ha cuadruplicado en los últimos ciento diez años. Han sido estas capitales focos de atracción provincial.

La evolución registrada por cada una de estas capitales ha seguido distinto ritmo. Badajoz se mantiene prácticamente estacionaria desde 1860 hasta 1900; la entrada del nuevo siglo representa para Badajoz un fuerte empuje demográfico, tras el cual crece moderadamente hasta 1930; en los decenios subsiguientes ya es franco el incremento de su población, en especial en el período 
1950-1960, en que se registra un incremento del 23 por 100; en el último decenio ha aumentado el número de sus habitantes en un 17 por 100.

Cáceres presenta una situación de débil aumento de población hasta 1910; en el decenio 1910-1920 registra un incremento del 10 por 100; en el decenio siguiente, al igual que en el período 19501960 , ha experimentado un aumento reducido; pero el gran aumento del número de habitantes se produjo en el decenio 1930-1940, con el 24 por 100, seguido del 10 por 100 en el decenio siguiente; el decenio último - 1960-1970 - registra un notable incremento del 14 por 100 . En cierto modo, pues, ambas ciudades difieren sustancialmente en su evolución demográfica.

\section{De los Partidos Judiciales}

Estudiamos la evolución de la población de los Partidos Judiciales de Badajoz y Cáceres solamente en lo que va de siglo, que supone un período de setenta años, suficientemente amplio para conocer claramente la tendencia del fenómeno.

En la tabla adjunta se detalla la población de cada Partido, según los censos de población elaborados en el presente siglo y los números índices obtenidos, tomando como base el año 1970.

C E N S O S

\begin{tabular}{|c|c|c|c|c|c|c|c|c|}
\hline Partidos Judiciales & 1900 & 1910 & 1920 & 1930 & 1940 & 1950 & 1960 & 1970 \\
\hline & \multicolumn{8}{|c|}{ CIFRAS ABSOLLUTAS } \\
\hline \multicolumn{9}{|l|}{ Provincia de Badajoz } \\
\hline Alburquerque $\ldots \ldots \ldots$ & 23.975 & 30.163 & 29.804 & 32.501 & 33.027 & 34.644 & 33.421 & 26.378 \\
\hline Almendralejo $\ldots . . . . .$. & 49.090 & 55.710 & 61 & 16 & 75 & & 38 & 63.935 \\
\hline $\begin{array}{lllll}\text { Badajoz } & \ldots & \ldots & \ldots & \ldots\end{array}$ & 34.268 & 38.814 & 42.810 & 49.854 & 61.585 & 86.152 & 104.478 & 109.044 \\
\hline Castuera ... & 37.607 & 46.499 & 51.124 & 58.435 & 58.555 & 65.065 & 61.723 & 43.563 \\
\hline Don Benito $\ldots \ldots \ldots$ & 29.735 & 34.265 & 37.725 & 39.341 & 38.605 & 43.289 & 47.247 & 45.815 \\
\hline Fregenal de la Sierra & 35.186 & 38.489 & 40.776 & 33.663 & 34.060 & 35.665 & 32.522 & 24.251 \\
\hline Fuente de Cantos ... & 35.734 & 40.078 & 44.570 & 45.898 & 47.169 & 48.213 & 43.841 & 31.758 \\
\hline Herrera del Duque ... & 21.604 & 24.451 & 26.036 & 30.088 & 32.121 & 35.845 & 37.005 & 25.849 \\
\hline Jerez de los Caballeros. & 38.484 & 41.045 & 48.969 & 51.932 & 54.440 & 58.181 & 56.131 & 38.019 \\
\hline Tereng & 52.992 & 57.966 & 65.356 & 70.916 & $70: 764$ & 74.216 & 67.985 & 44.561 \\
\hline Mérida & 48.209 & 57.170 & 62.134 & 71.340 & 82.691 & 87.813 & 106.492 & 97.418 \\
\hline Olivenza & 31.24 & & 38.400 & 40.726 & 41.823 & 44.568 & & 30.153 \\
\hline Puebla de Alcocer & 20.647 & 23.6 & 25.211 & 28.107 & 27.750 & 31.765 & 31.966 & 23.083 \\
\hline Villanueva de la Serena. & 27. & 32 & 32.373 & 34.702 & 35.045 & 38.333 & 41.500 & 39.237 \\
\hline & 33.692 & $35: 849$ & 38.164 & 47.199 & 49.179 & 52.033 & 53.365 & 44.535 \\
\hline
\end{tabular}




\begin{tabular}{|c|c|c|c|c|c|c|c|c|}
\hline Partidos Judiciales & 1900 & 1910 & 1920 & 1930 & 1940 & 1950 & 1960 & 1970 \\
\hline & \multicolumn{8}{|c|}{ CIFRAS ABSOLUTAS } \\
\hline \multicolumn{9}{|l|}{ Provincia de Cáceres } \\
\hline Alcántara & 20.089 & 21.251 & 21.391 & - & 24.620 & 24.233 & 21.309 & 17.593 \\
\hline iceres & 39.967 & 42.060 & 50.233 & 56.244 & 73.300 & 80.348 & 80.722 & 98.886 \\
\hline $\begin{array}{lllll}. . & \ldots & \ldots & \ldots\end{array}$ & 23.703 & 23.613 & 22.817 & 34.370 & 27.655 & 31.135 & 38.164 & 34.927 \\
\hline $\begin{array}{llll}\text { Garrovillas } & \ldots & \ldots & \ldots\end{array}$ & 21.201 & 23.8 & 23.605 & 38.116 & 27. & 27 & 52 & 16.874 \\
\hline Hervás ... ... & 30.591 & 33.253 & 32.076 & 34.196 & 37.412 & 39.339 & 39.382 & 33.321 \\
\hline Hoyos ...... & 23.341 & 25.651 & 23.671 & 26.342 & 30 & 31.211 & 28. & 22.782 \\
\hline $\begin{array}{llllll} & \\
\text { Jarandilla } & \ldots & \ldots & \ldots & \ldots & \ldots\end{array}$ & 23.826 & 28.162 & 27.709 & 27.144 & 31.804 & 35.189 & 38.461 & 31.412 \\
\hline Logrosán ... & 28.701 & 32.829 & 34.056 & 36.360 & 39.189 & 42.968 & 38.887 & 26.859 \\
\hline $\begin{array}{llll}\text { Montánchez } & \ldots & \ldots & \ldots\end{array}$ & 24.755 & 27. & 27. & 29. & 33. & 34. & 3 & 21.284 \\
\hline Navalmoral de la Mata. & 30.058 & 33.604 & 33.274 & 36.352 & 40.223 & 44.413 & 48.451 & 37.003 \\
\hline $\begin{array}{cccccc}\text { Plasencia } & \ldots & \ldots & \ldots & \ldots & \ldots\end{array}$ & 33.764 & 38. & 41.107 & 50.272 & & 68.224 & 73.443 & 73.386 \\
\hline $\begin{array}{lllll}\ldots & \ldots & \ldots & \ldots\end{array}$ & 43 & 47.135 & 49.621 & 56.488 & 60.484 & 62.696 & 57.872 & 24.258 \\
\hline Valencia de & 18.189 & 26 & 22.561 & 24.015 & 27.165 & 27.484 & 24.400 & 16.586 \\
\hline
\end{tabular}

NUMEROS INDICES. BASE $1970=100$

\begin{tabular}{|c|c|c|c|c|c|c|c|c|c|}
\hline \multicolumn{10}{|l|}{ Provincia de Badajoz } \\
\hline Alburquerque $\quad \ldots \quad \ldots$ & ... & 90,9 & 114,3 & 113,0 & 123,2 & 125,2 & 131,3 & 126,7 . & 100 \\
\hline Almendralejo $\ldots \ldots$ & $\ldots$ & 76,8 & 87,1 & 95,7 & 105,9 & 118,4 & 125,1 & 116,1 & 100 \\
\hline $\begin{array}{ccccc}\text { Badajoz } & \ldots & \ldots & \ldots & \ldots\end{array}$ & $\ldots$ & 31,4 & 35,6 & 39,3 & 45,7 & 56,5 & 79,0 & 95,8 & 100 \\
\hline $\begin{array}{llllll}\text { Castuera } & \ldots & \ldots & \ldots & \ldots\end{array}$ & $\ldots$ & 86,3 & 106,7 & 117,3 & 134,1 & 134,4 & 149,4 & 141,6 & 100 \\
\hline Don Benito $\ldots . . .$. & $\ldots$ & 65,1 & 74,8 & 82,4 & 85,9 & 84,3 & 94,5 & 103,1 & 160 \\
\hline Fregenal de la Sierra & $\ldots$ & 145,1 & 158,7 & 168,1 & 138,8 & 147,9 & 147,1 & 134,1 & 100 \\
\hline Fuente de Cantos ... & $\ldots$ & 112,5 & 126,2 & 140,3 & 144,5 & 148,5 & 151,8 & 138,0 & 100 \\
\hline Herrera del Duque & $\ldots$ & 83,6 & 94,6 & 100,7 & 116,4 & 124,3 & 148,7 & 143,2 & 100 \\
\hline Jerez de los Caballero & & 101,2 & 108,0 & 128,8 & 136,6 & 143,2 & 153,0 & 147,6 & 160 \\
\hline $\begin{array}{lllll}\text { Llerena } & \ldots & \ldots & \ldots & \ldots\end{array}$ & $\ldots$ & 118,9 & 130,1 & 146,7 & 159,2 & 158,8 & 165,5 & 152,6 & 100 \\
\hline $\begin{array}{lllllll}\text { Mérida } & \ldots & \ldots & \ldots & \ldots & \ldots\end{array}$ & $\ldots$ & 49,5 & 58,7 & 63,8 & 73,2 & 84,9 & 90,1 & 109,3 & 160 \\
\hline $\begin{array}{lllll}\text { Olivenza } & \ldots & \ldots & \ldots & \ldots\end{array}$ & $\ldots$ & 103,7 & 121,7 & 127,3 & 135,6 & 138,8 & 137,9 & 140,8 & 100 \\
\hline Puebla de Alcocer & $\ldots$ & 89,4 & 102,4 & 109,2 & 121,8 & 120,2 & 137,6 & 138,5 & 100 \\
\hline Villanueva de la Seren & & 70,8 & 82,5 & 82,5 & 88,4 & 89,3 & 97,6 & 105,8 & 100 \\
\hline $\begin{array}{llllll}\text { Zafra } & \ldots & \ldots & \ldots & \ldots & \ldots\end{array}$ & $\cdots$ & 75,7 & 80,5 & 85,7 & 106,0 & 110,5 & 116,8 & 119,8 & 100 \\
\hline \multicolumn{10}{|l|}{ Provincia de Cáceres } \\
\hline 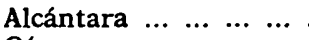 & ... & 114,1 & 120,7 & 121,6 & - & 139,9 & 137,7 & 121,1 & 100 \\
\hline Cáceres .. & $\ldots$ & 40,4 & 42,5 & 50,8 & 56,9 & 74,1 & 81,2 & 31,6 & 100 \\
\hline $\begin{array}{llll}\ldots & \ldots & \ldots & \ldots\end{array}$ & $\ldots$ & 67,9 & 67,6 & 65,3 & 98,3 & 79,2 & 89,1 & 109,3 & 100 \\
\hline Garrovillas & $\ldots$ & 125,6 & 141,2 & 139,9 & 225,9 & 162,0 & 164,2 & 145,0 & 100 \\
\hline $\begin{array}{lllll}\text { Hervás } & \ldots & \ldots & \ldots & \ldots\end{array}$ & $\ldots$ & 91,8 & 99,8 & 96,3 & 102,6 & 112,3 & 118,1 & 118,2 & 100 \\
\hline $\begin{array}{llllll}\text { Hoyos } & \ldots & \ldots & \ldots & \ldots & \ldots\end{array}$ & $\ldots$ & 102,5 & 112,6 & 104,3 & 115,6 & 129,1 & 137,0 & 127,1 & 130 \\
\hline Jarandilla $\ldots$ & & 75,8 & 89,7 & 88,2 & 86,4 & 101,2 & 112,0 & 122,4 & 100 \\
\hline $\begin{array}{llllll}\text { Logrosán } & \ldots & \ldots & \ldots & \ldots\end{array}$ & $\ldots$ & 106,5 & 122,3 & 126,8 & 135,4 & 145,9 & 160,0 & 144,8 & 100 \\
\hline $\begin{array}{llll}\text { Montánchez } & \ldots & \ldots & \ldots\end{array}$ & $\ldots$ & 116,3 & 128,3 & 131,1 & 140,2 & 151,8 & 160,3 & 141,0 & 100 \\
\hline Navalmoral de la Mat & & 81,2 & 90,8 & 89,9 & 98,2 & 108,7 & 120,0 & 130,9 & 100 \\
\hline $\begin{array}{lllll}\text { Plasencia } & \ldots & \ldots & \ldots & \ldots\end{array}$ & $\ldots$ & 46,0 & 53,1 & 56,0 & 68,5 & 81,0 & 93,0 & 100,1 & 100 \\
\hline $\begin{array}{ccccc}\text { Trujillo } & \ldots & \ldots & \ldots & \ldots\end{array}$ & $\ldots$ & 181,3 & 194,3 & 204,5 & 232,9 & 249,3 & 258,5 & 238,2 & 100 \\
\hline Valencia de Alcántar & & 109,7 & 121,3 & 136,0 & 144,8 & 163,8 & 165,7 & 147,1 & 100 \\
\hline
\end{tabular}

De la observación de dicha tabla se deduce, en primer lugar, una generalización del fenómeno de la disminución de la población en 1970, con respecto a 1960, en todos los Partidos Judiciales de ambas Provincias, a excepción de los dos Partidos de las respectivas capi- 
tales de Provincia. Estos últimos registran un incremento de pobıación con una tendencia creciente paulatina, que lleva a Badajoz a triplicar el número de sus habitantes en setenta años, y a Cáceres a registrar un aumento del 60 por 100 en el mismo período.

En segundo lugar, en la Provincia de Badajoz, los Partidos de Mérida, Don Benito y Villanueva de la Serena registran un aumento creciente de población en los setenta años del presente siglo, sin llegar a duplicarla, salvo Mérida, que presenta una disminución de población en 1970 que en ningún caso rebasa el 10 por 100 de la de 1960. Si estos tres Partidos disponen de zonas regables del Guadiana, también en la Provincia de Cáceres están los Partidos de Coria, Plasencia, Jarandilla y Navalmoral de la Mata, con zonas regables por el Tajo y sus afluentes Alagón, Jerte y Tiétar; estos Partidos duplicaron su población a lo largo del período consideradc, y también experimentan en 1970 disminuciones del 20 y 30 por 100 en la población de Jarandilla y Navalmoral de la Mata, y más débiles en Coria y Plasencia, con respecto a 1960.

En tercer término, podemos considerar el grupo de los 19 restantes Partidos Judiciales de ambas Provincias; se da el caso de que once de ellos - cinco en Badajoz y seis en Cáceres- tenían en 1900 más habitantes que en 1970, y siguieron una tendencia creciente junto con los demás hasta 1960, excepción hecha de Alcántara, que presenta sólo un ligero incremento, y Fregenal de la Sierra, que mantiene una situación estacionaria a lo largo del períocio. Todos ellos han registrado un fuerte descenso de población en el decenio 1960-1970, del orden del 40 al 60 por 100 en la mayor parte de los casos. La máxima disminución corresponde a Trujillo, con una pérdida del 138 por 100 de su población en diez años.

Ello corrobora la afirmación inicial de que la disminución de la población de Extremadura se ha extendido a toda la Región, salvo en las capitales y en sus respectivos Partidos Judiciales.

\section{EL CRECIMIENTO VEGETATIVO Y EL MOVIMIENTO MIGRATORIO}

El crecimiento vegetativo de Extremadura - la simple diferencia entre los nacidos y los fallecidos- da una tasa notable; a 911.910 asciende el incremento natural de la población extremeña en los 
setenta primeros años del presente siglo, de los que 538.427 corresponden a Badajoz y 373.483 a Cáceres.

El detalle por decenios es éste:

\begin{tabular}{|c|c|c|c|}
\hline & Extremadura & Badajoz & Cáceres \\
\hline $\begin{array}{cccccccccccc}\text { De } & 1901 & \text { a } & 1910 & \ldots & \ldots & \ldots & \ldots & \ldots & \ldots & \ldots & \ldots \\
\text { De } & 1911 & \text { a } & 1920 & \ldots & \ldots & \ldots & \ldots & \ldots & \ldots & \ldots & \ldots \\
\text { De } & 1921 & \text { a } & 1930 & \ldots & \ldots & \ldots & \ldots & \ldots & \ldots & \ldots & \ldots \\
\text { De } & 1931 & \text { a } & 1940 & \ldots & \ldots & \ldots & \ldots & \ldots & \ldots & \ldots & \ldots \\
\text { De } & 1941 & \text { a } & 1950 & \ldots & \ldots & \ldots & \ldots & \ldots & \ldots & \ldots & \ldots \\
\text { De } & 1951 & \text { a } & 1960 & \ldots & \ldots & \ldots & \ldots & \ldots & \ldots & \ldots & \ldots \\
\text { De } & 1961 & \text { a } & 1970 & \ldots & \ldots & \ldots & \ldots & \ldots & \ldots & \ldots & \ldots \\
\end{array}$ & $\begin{array}{r}107.718 \\
77.561 \\
126.029 \\
118.515 \\
137.940 \\
188.521 \\
155.626\end{array}$ & $\begin{array}{r}62.754 \\
52.436 \\
73.605 \\
67.254 \\
77.384 \\
111.820 \\
93.174\end{array}$ & $\begin{array}{l}44.964 \\
25.125 \\
52.424 \\
51.261 \\
60.556 \\
76.701 \\
62.452\end{array}$ \\
\hline
\end{tabular}

Los dos períodos de disminución del crecimiento vegetativo obedecen: el primero, a la mortalidad registrada durante la epidemia del año 1918; el segundo, a la derivada de nuestra contienda civil. La tendencia ascendente de la tasa de natalidad a partir de 1951 justifica el incremento del crecimiento vegetativo en el decenio 19501960; el descenso de población en el decenio siguiente explica la disminución del crecimiento vegetativo en dicho período.

Los cálculos del movimiento migratorio se han hecho teniendo solamente en cuenta la población existente en los momentos inicial y final de cada período y el flujo de nacimientos y de defunciones entre dichos momentos para deducir el saldo migratorio de cada decenio. Se expresan en las siguientes cifras:

\begin{tabular}{|c|c|c|c|}
\hline & Extremadura & Badajoz & Cáceres \\
\hline $\begin{array}{lllllllllll}\text { De } 1901 & 19 & 1910 & \ldots & \ldots & \ldots & \ldots & \ldots & \ldots & \ldots \\
\text { De } & 1911 & \text { a } & 1920 & \ldots & \ldots & \ldots & \ldots & \ldots & \ldots & \ldots \\
\text { De } 1921 & \text { a } & 1930 & \ldots & \ldots & \ldots & \ldots & \ldots & \ldots & \ldots \\
\text { De } & 1931 & \text { a } & 1940 & \ldots & \ldots & \ldots & \ldots & \ldots & \ldots & \ldots \\
\text { De } & 1941 & \text { a } & 1950 & \ldots & \ldots & \ldots & \ldots & \ldots & \ldots & \ldots \\
\text { De } & 1951 & \text { a } & 1960 & \ldots & \ldots & \ldots & \ldots & \ldots & \ldots & \ldots \\
\text { De } & 1961 & \text { a } & 1970 & \ldots & \ldots & \ldots & \ldots & \ldots & \ldots & \ldots\end{array}$ & 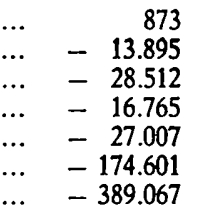 & $\begin{array}{rr} & 10.206 \\
- & 1.017 \\
- & 15.812 \\
- & 27.125 \\
- & 4.151 \\
- & 93.230 \\
- & 239.985\end{array}$ & $\begin{array}{l}-\quad 9.333 \\
=\quad 12.878 \\
-\quad 12.700 \\
-\quad 10.360 \\
-\quad 22.856 \\
-\quad 81.371 \\
-149.082\end{array}$ \\
\hline $\begin{array}{lllllllll}\text { Totales } & \ldots & \ldots & \ldots & \ldots & \ldots & \ldots & \ldots\end{array}$ & $\ldots \quad-648.974$ & -371.114 & -277.860 \\
\hline
\end{tabular}

Del cuadro anterior se deduce, en primer término, que durante los primeros setenta años del presente siglo, Extremadura ha perdido casi 650.000 habitantes, lo que equivale a algo más del 70 por 100 de su crecimiento vegetativo. Para Cáceres esta pérdida se eleva al 74 por 100, mientras que con respecto a Badajoz no llega al 70 .

En el primer decenio, Extremadura registra una inmigración neta 
casi simbólica, manifestada más claramente en Badajoz y casi contrarrestada por la emigración cacereña; para la Región en su conjunto, la emigración se manifiesta viva en el decenio siguiente, y se duplica en el de 1921-1930 por la extensión del fenómeno a Badajoz. En el período 1931-1940 las posiciones de ambas Provincias resultan antagónicas, pues frente a la fuerte emigración de Badajoz se observa un aumento de población de Cáceres que, sin embargo, sufre una pérdida importante de habitantes, muy atenuada en Badajoz, en el período de 1941 a 1950. A partir de este último año se generaliza el fenómeno migratorio, con cifras muy superiores a las de los decenios anteriores, llegando casi a absorber el crecimiento vegetativo en los años 1951 a 1960 para el conjunto de Extremadura, y lo absorbe realmente con respecto a la Provincia de Cáceres; y se acrecienta en el último decenio, con la pérdida de casi 400.000 habitantes, incluida la totalidad del crecimiento vegetativo.

De esta forma se explica el fuerte decrecimiento - de alrededor del 20 por 100 - de la población extremeña, a que nos hemos referido en el apartado anterior.

\section{LA EVOLUCION DE LA POBLACION ACTIVA}

\section{EN SU CONJUNTO}

En uno de los trabajos que comentamos se ha estudiado la evolución de la población activa extremeña en un período de dieciséis años, que se extiende de 1955 a 1971; nosotros nos limitaremos a considerarlo sólo en cinco momentos, a los que corresponden estas cifras:

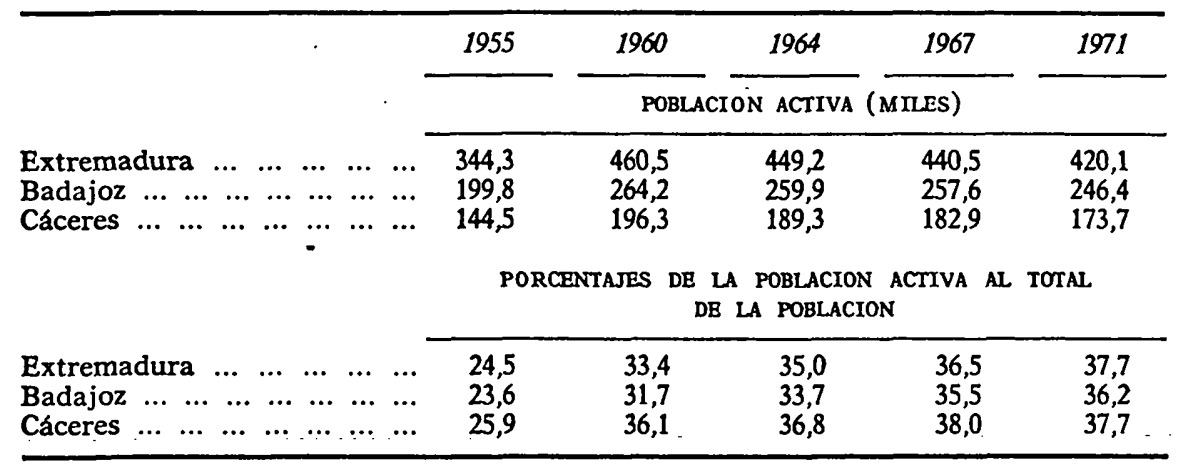


En este período, mientras la población española se había incrementado en un 17 por 100, la extremeña había disminuido en algo más del 20, lo que lógicamente había de influir en el volumen de la población activa, que se mantiene in crescendo en cifras absolutas, si bien a partir de 1962 se inicia un proceso decreciente; pero en cifras relativas, la población activa aumenta, lo que se explica por la paulatina incorporación de la mujer a la mano de obra, en especial de los trabajos en las industrias de transformación de los productos del campo.

\section{PoR SECTORES DE LA ACTIVIDAD ECONÓMICA}

La distribución de la población activa extremeña, por sectores de la actividad económica en el período considerado, es ésta:

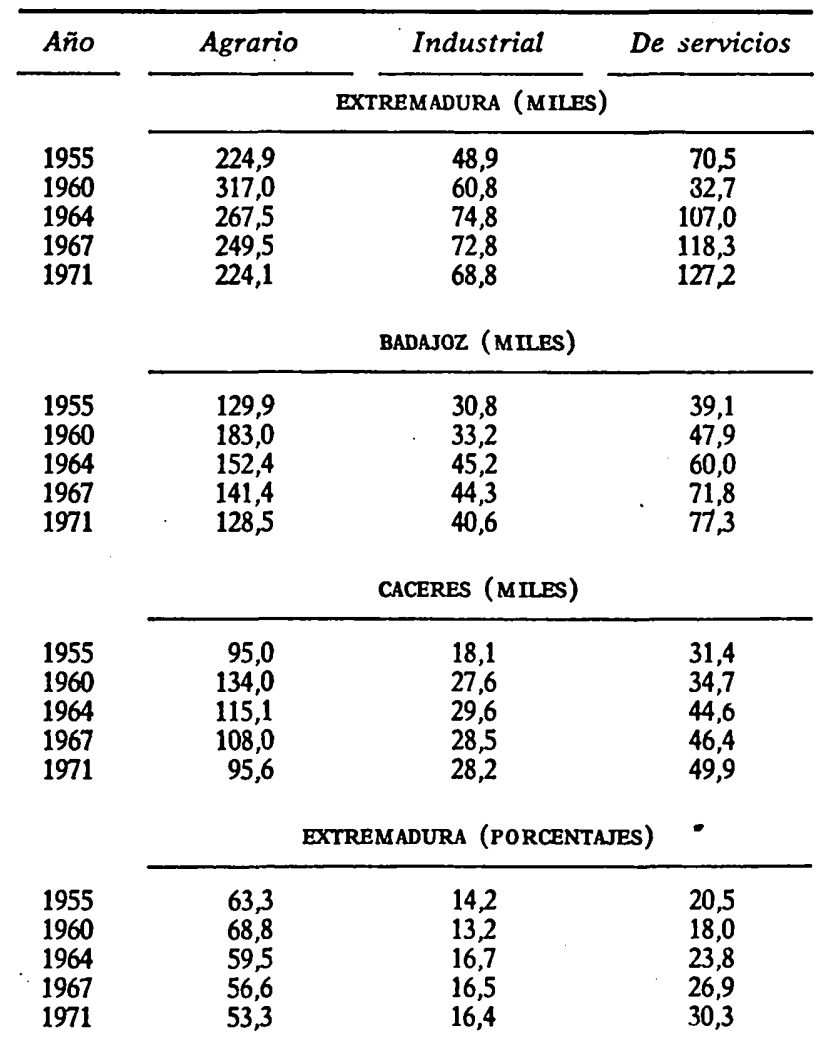


ESTADISTICA

\begin{tabular}{|c|c|c|c|}
\hline$A \bar{n} o$ & Agrario & Industrial & De servicios \\
\hline & \multicolumn{3}{|c|}{ BADAJOZ (PORCENTAJES) } \\
\hline $\begin{array}{l}1955 \\
1960 \\
1964 \\
1967 \\
1971\end{array}$ & $\begin{array}{l}65,0 \\
69,3 \\
58,6 \\
54,9 \\
52,1\end{array}$ & $\begin{array}{l}15,4 \\
12,6 \\
17,4 \\
17,2 \\
16,5\end{array}$ & $\begin{array}{l}19,6 \\
18,1 \\
24,0 \\
27,9 \\
31,4\end{array}$ \\
\hline 1971 & \multicolumn{3}{|c|}{ CACERES (PORCENTAJES) } \\
\hline $\begin{array}{l}1955 \\
1960 \\
1964 \\
1967 \\
1971\end{array}$ & $\begin{array}{l}65,8 \\
68,2 \\
60,8 \\
59,0 \\
55,0\end{array}$ & $\begin{array}{l}12,5 \\
14,1 \\
15,6 \\
15,6 \\
16,3\end{array}$ & $\begin{array}{l}21,7 \\
17,7 \\
23,6 \\
25,4 \\
28,7\end{array}$ \\
\hline
\end{tabular}

La distribución sectorial de la población activa extremeña manifiesta el estado de subdesarrollo en que se halla esta Región. Aunque la población agraria ha descendido a partir de 1962, se mantiene en cotas todavía muy altas. La mano de obra industrial se ha mantenido prácticamente estacionaria después del incremento registrado en 1964, y ha sido el sector de servicios el que presenta un incremento más acusado, tanto en el conjunto de la Región como en ambas Provincias.

Si se compara esta distribución con la de la población activa total de España, destaca más acentuadamente la lentitud con que se produce el desarrollo en Extremadura, y la distancia que le separa del de otras Regiones. La población activa agraria de España había disminuido, en el mismo período, del 37,8 al 28,1 por ciento: la industrial se había incrementado del 30 al 34,4 por 100; y la de servicios, del 32,3 al 37,5 .

\section{LA EVOLUCION DE LA RENTA}

\section{Total}

La renta total, en los años considerados, se cifraba en millones de pesetas de cada año, así:

\begin{tabular}{|c|c|c|c|}
\hline Año & Extremadura & Badajoz & Cáceres \\
\hline $\begin{array}{l}1955 \\
1960 \\
1964 \\
1967 \\
1971\end{array}$ & $\begin{array}{r}8.827,9 \\
15.738,1 \\
21.184,1 \\
32.541,2 \\
47.496,0\end{array}$ & $\begin{array}{r}5.376,1 \\
10.029,6 \\
12.753,9 \\
20.212,8 \\
29.833,0\end{array}$ & $\begin{array}{r}3.451,8 \\
5.708,5 \\
8.430,7 \\
12.328,4 \\
17.663,0\end{array}$ \\
\hline
\end{tabular}


Que en pesetas constantes de 1970 son:

\begin{tabular}{|c|c|c|c|}
\hline$A \tilde{n} o$ & Extremadura & Badajoz & Cáceres \\
\hline $\begin{array}{l}1955 \\
1960 \\
1964 \\
1967 \\
1971\end{array}$ & $\begin{array}{l}23.367,5 \\
28.611,9 \\
30.717,7 \\
36.869,2 \\
43.886,3\end{array}$ & $\begin{array}{l}14.230,6 \\
18.233,8 \\
18.493,2 \\
22.901,1 \\
27.565,7\end{array}$ & $\begin{array}{r}9.136,9 \\
10.378,1 \\
12.224,5 \\
14.968,1 \\
16.320,6\end{array}$ \\
\hline
\end{tabular}

Cuyos índices reales de elevación de rentas sobre 1955 son:

\begin{tabular}{|c|c|c|c|}
\hline Año & Extremadura & Badajoz & Cáceres \\
\hline $\begin{array}{l}1960 \\
1964 \\
1967 \\
1971\end{array}$ & $\begin{array}{l}22,4 \\
31,5 \\
57,8 \\
87,8\end{array}$ & $\begin{array}{l}28,1 \\
29,9 \\
60,9 \\
93,7\end{array}$ & $\begin{array}{l}13,6 \\
33,8 \\
52,9 \\
78,6\end{array}$ \\
\hline
\end{tabular}

Mientras la renta nacional española pasó del 15,1 en 1960 al 156,1, es decir, se multiplicó por diez, la extremeña se multiplicó por cuatro, ofreciendo un mayor ritmo de crecimiento Cáceres que Badajoz.

\section{POR HABITANTE}

Uno de los índices económicos más representativos que se utilizan para determinar el nivel de desarrollo de una Región es la renta por habitante. Pese a las limitaciones que tiene, los economistas lo consideran instrumento indispensable para conocer la evolución económica de un país o de una Región.

La renta por habitante, cociente resultante de dividir la renta total de un país o Región por el número de sus habitantes, depende, pues, de dos factores, y la variación de cada uno de ellos puede modificar el cociente, bien por incremento de la renta, bien por disminución de la población. Otro factor a tener en cuenta es la depreciación de la moneda; de ahí la conveniencia de operar con pesetas constantes, además de hacerlo con pesetas nominales de cada año. Otro factor influyente lo constituyen las remesas de los emigrantes, de las que Extremadura es receptora.

La renta por habitante ha sido la siguiente en los años de referencia, según los estudios del Banco de Bilbao: 
ESTADISTICA

\begin{tabular}{|c|c|c|}
\hline$A \bar{n} o$ & Badajoz & Cáceres \\
\hline \multirow{3}{*}{$\begin{array}{l}1955 \\
1960 \\
1964 \\
1967 \\
1971\end{array}$} & \multicolumn{2}{|c|}{ EN PESETAS DE CADA AÑO } \\
\hline & $\begin{array}{r}6.361 \\
12.021 \\
16.553 \\
27.900 \\
43.846\end{array}$ & $\begin{array}{r}5.193 \\
10.486 \\
16.397 \\
25.611 \\
38.953\end{array}$ \\
\hline & \multicolumn{2}{|c|}{ EN PESETAS DE 1970} \\
\hline $\begin{array}{l}1955 \\
1960 \\
1964 \\
1967 \\
1971\end{array}$ & $\begin{array}{l}16.838 \\
21.854 \\
21.204 \\
31.611 \\
40.514\end{array}$ & $\begin{array}{l}16.393 \\
19.064 \\
21.005 \\
29.017 \\
35.993\end{array}$ \\
\hline
\end{tabular}

Como vemos, la renta por habitante en las dos Provincias extremeñas se ha duplicado en los dieciséis años considerados; pero, sin embargo, su diferencia con la renta promedia nacional es cada vez mayor, es decir, que las dos Provincias van quedando rezagadas en relación con el resto de España.

No sólo se trata, pues, de una de las Regiones más subdesarrolladas de España, sino que continuará siéndolo si la Administración no se decide a fomentar la puesta en producción de los recursos que posee esta Región, actualmente sin explotar o mal explotados, para incrementar su renta y aminorar su diferencia con el resto de la Nación, la España desarrollada. Para ello es necesario promover, mediante inversiones adecuadas, un relanzamiento de la economía de Extremadura, que tienda a equilibrarla con las demás para lo grar una igualdad en el nivel de vida y de bienestar de todas las Regiones españolas. 
REVL-1975, núm. 185. BALLESTER ROS, IGNACIO. LA EVOLUCION DE LA POBLACION Y DE L... REVL-1975, núm. 185. BALLESTER ROS, IGNACIO. LA EVOLUCION DE LA POBLACION Y DE L... 


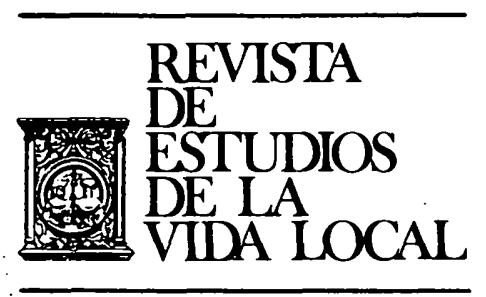

\section{JURISPRUDENCIA}


REVL-1975, núm. 185. BALLESTER ROS; IGNACIO. LA EVOLUCION DE LA POBLACION Y DE L... 